\title{
Enucleação como alternativa de tratamento cirúrgico para o adenoma pleomórfico: relato de caso
}

Enucleation as an alternative surgical treatment for pleomorphic adenoma: case report

Enucleación como tratamiento quirúrgico alternativo para el adenoma pleomórfico: reporte de caso

\section{Rodrigo dos Santos PEREIRA ${ }^{1}$ \\ Jonathan Ribeiro da SILVA ${ }^{2}$ \\ Raphael Capelli GUERRA ${ }^{3}$}

'Cirurgião Bucomaxilofacial - HGNI, Mestre e Doutor em Odontologia - UNESP, Pós doutorado - UNESP, Fellow AO - Universidade de Hamburgo, Alemanha, Professor Cirurgia e Traumatologia Bucomaxilofacial - HCT-CO / UNIFESO, Professor Pós-Graduação stricto sensu em Odontologia - UNIGRANRIO

${ }^{2}$ Especialista em Cirurgia e Traumatologia Bucomaxilofacial, Mestre em Odontologia pela UNIGRANRIO Doutor em Odontologia pela Faculdade de Odontologia de Araçatuba - UNESP

Professor de Cirurgia Bucomaxilofacial do Centro Universitário Serra dos Órgãos - UNIFESO

${ }^{3}$ Especialista em Cirurgia e Traumatologia Bucomaxilofacial, Mestre em Oncologia - Hospital AC Camargo, Chefe de Equipe de Cirurgia e Traumatologia Bucomaxilofacial do Hospital Leforte. São Paulo - São Paulo, Brasil Doutorando em Odontologia pela Faculdade de Odontologia de Araçatuba - UNESP

\section{Resumo}

O adenoma pleomórfico é um tumor benigno que acomete as glândulas salivares tendo maior incidência na glândula parótida. Seu tratamento é motivo de discussão entre os profissionais devido a sua particularidade na abordagem de acordo com o nervo facial. Este relato de caso demonstra a enucleação como uma técnica cirúrgica conservadora que buscou remover o tumor, através de um acesso retromandibular, visando reduzir complicações transcirúgicas e pós-operatórias.

Descritores: Adenoma Pleomorfo; Cirurgia Bucal; Glândulas Salivares.

\section{Abstract}

Pleomorphic adenoma is a benign tumor that affects the salivary glands with higher incidence in the parotid gland. The treatment has been motiving for discussion among professionals due to its particularity and the proximity with facial nerve. The aim of this case report, is demonstrate the enucleation as a conservative surgical technique that sought to remove the tumor through a retromandibular approach, aiming to reduce postoperative and trans-surgical complications.

Descriptors: Adenoma, Pleomorphic; Surgery, Oral; Salivary Glands.

\section{Resumen}

El adenoma pleomórfico es un tumor benigno que afecta las glándulas salivales y tiene una mayor incidencia en la glándula parótida. Su tratamiento es motivo de discusión entre profesionales debido a su particularidad en el abordaje según el nervio facial. Este informe de caso demuestra la enucleación como una técnica quirúrgica conservadora que buscaba extirpar el tumor mediante un abordaje retromandibular, con el objetivo de reducir las complicaciones posoperatorias y transquirúrgicas.

Descriptores: Adenoma Pleomórfico; Cirugía Bucal; Glándulas Salivales.

\section{INTRODUÇÃO}

O adenoma pleomórfico é o tumor mais acometido nas glândulas salivares ${ }^{1}$, com cerca de 70 a $90 \%$ de incidência sendo destes $80 \%$ em glândulas parótidas $^{2,3}$. Acomete pessoas de várias idades, de recém-nascidos a idosos, com prevalência pelo gênero feminino e geralmente entre a quarta e sexta décadas de vida ${ }^{4}$. Sua etiologia é controversa, sendo constituído de elementos epiteliais e mesenquimais. Seu componente epitelial é diverso, contendo combinações de células ductais, células escamosas, células claras, células fusiformes, células oncocíticas e mucosas. Podem ser inteiramente ou parcialmente encapsulados ou até mesmo diretamente no parênquima da glândula salivar ${ }^{5}$. Projeções digitiformes do tumor através da cápsula não apresentam significado diagnóstico, porém sua presença parece aumentar o risco de recorrência do tumor $^{6}$.

Os adenomas pleomórficos que afetam a glândula parótida se apresentam clinicamente como lesões indolores, nodulares, de crescimento lento, com mobilidade e de limites bem definidos ${ }^{7}$. O local de maior incidência deste tumor na glândula é o lobo superficial principalmente nas regiões pré-auricular e retromandibular sendo ambos os lados afetados em proporções idênticas ${ }^{2}$. Noventa porcento dessas neoplasias, estão localizadas no lobo superficial da glândula, lateralmente ao nervo facial ${ }^{8}$.
A remoção dos tumores benignos da glândula

parótida é um assunto de grande controvérsia, pois o nervo facial encontra-se em íntima relação com quase todos os tumores benignos da glândula. Neste caso, sempre há a questão quanto a forma radical de excisão da lesão. Muitos cirurgiões defendem a forma de procedimentos radicais, com alta possibilidade de lesão ao nervo facial, tendo a suposição de que desta forma há redução das elevadas taxas de recorrências ${ }^{2}$.

Há três formas de procedimentos cirúrgicos para o tratamento do adenoma pleomórfico na glândula parótida: A enucleação, a parotidectomia superficial e a parotidectomia total ${ }^{9}$. A primeira consiste em remover o tumor sem a remoção de tecido glandular adjacente. A segunda propõe remover o tumor com parte da glândula aderida ao mesmo. E a terceira a glândula parótida é totalmente removida com a preservação do nervo facial.

O objetivo deste artigo é demonstrar a enucleação como um tratamento conservador e viável para remoção deste tipo de lesão com ênfase na importância do diagnóstico pré-cirúrgico.

\section{CASO CLÍNICO}

Paciente do sexo masculino, 34 anos, melanoderma, procurou o Serviço de Cirurgia Bucomaxilofacial do Hospital Geral de Nova Iguaçu no Rio de Janeiro, queixando-se de aumento de 
volume na hemiface direita, com crescimento lento e progressivo em um período de 3 anos. Ao exame clínico, evidenciou-se uma lesão nodular, de consistência firme, com mobilidade, com limites bem definidos, localizada na região retromandibular, no lado direito, indolor a palpação (Figura 1).

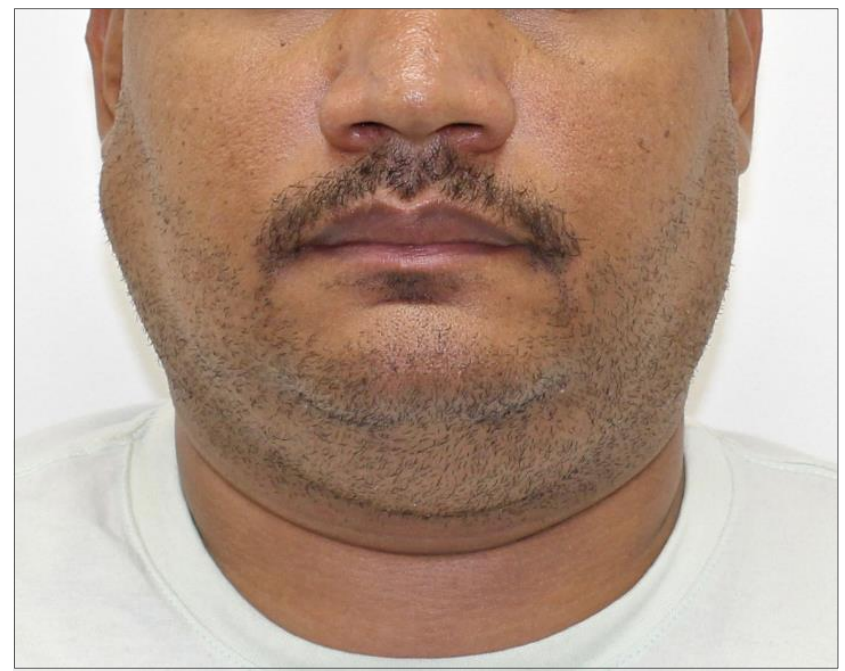

Figura 1: Imagem clínica evidenciando aumento de volume na região parotídea direita.

Foi realizado exame tomográfico que evidenciou lesão nodular, circunscrita e superficialmente à glândula parótida direita (Figura 2).

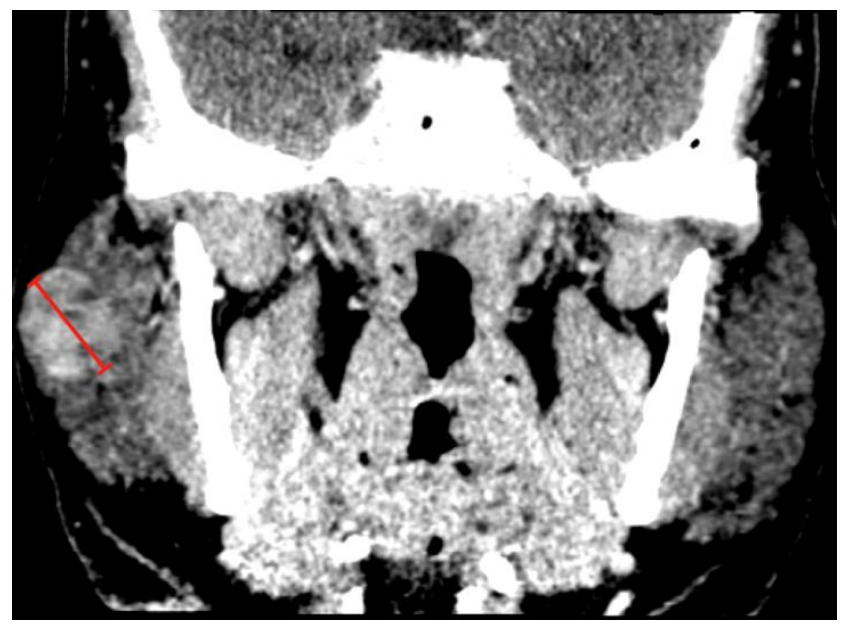

Figura 2: Imagem do corte coronal da tomografia computadorizada evidenciando a lesão na glândula parótida direita.

Em virtude das dimensões da lesão, optou-se pela realização de biópsia incisional através de uma core biopsia. O tecido obtido foi encaminhado ao serviço de anatomia patológica e o diagnóstico de adenoma pleomórfico foi confirmado. Tendo em vista essas características, o tratamento cirúrgico de escolha foi a enucleação do tumor. Exames laboratoriais de rotina foram solicitados para avaliar as condições sistêmicas do paciente, e evidenciou-se ausência de patologias associadas. O paciente foi submetido ao centro cirúrgico para realização do procedimento cirúrgico sob anestesia geral com entubação orotraqueal. Através do planejamento proposto uma incisão retromandibular de $4 \mathrm{~cm}$ foi feita e dissecou-se os tecidos subcutâneos até o encontro do músculo platisma. Para ultrapassá-lo, optou-se por uma dissecção romba com uma tesoura Metzenbaum até a cápsula da glândula parótida direita. Do mesmo modo dissecou-se a cápsula até a exposição da lesão (Figura 3).

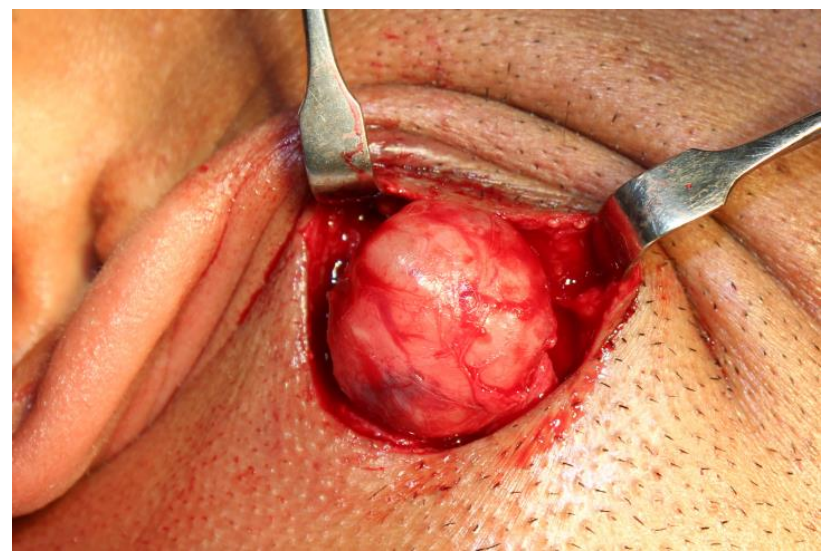

Figura 3: Fotografia transcirúrgica evidenciando o adenoma pleomórfico in situ.

Após, o tumor foi enucleado cuidadosamente para não haver rompimento e não deixar resíduos na glândula (Figura 4). Os planos internos foram suturados com fio reabsorvível de ácido poliglicólico 3-0 e a pele foi suturada com nylon monofilamentado 4-0. A peça cirúrgica foi enviada para o serviço de anatomia patológica onde pode se evidenciar a lesão por completo (Figura 5).

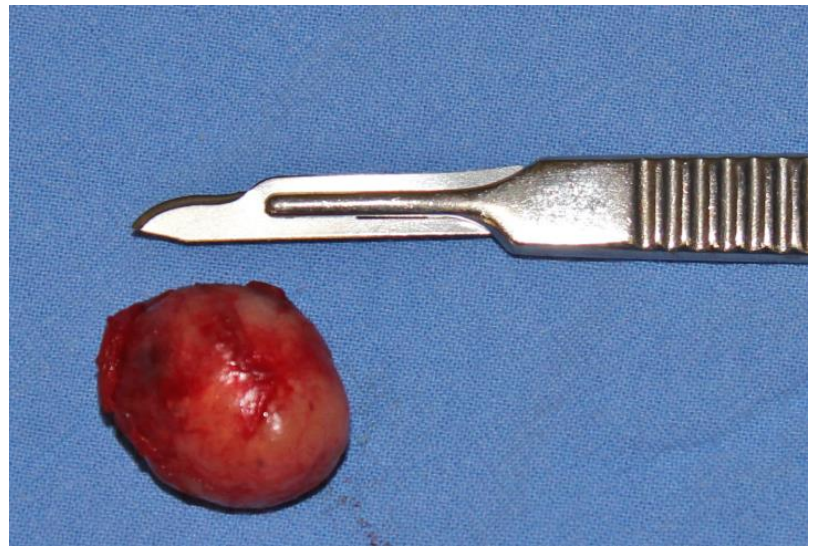

Figura 4: Fotografia da lesão pós-enucleação.

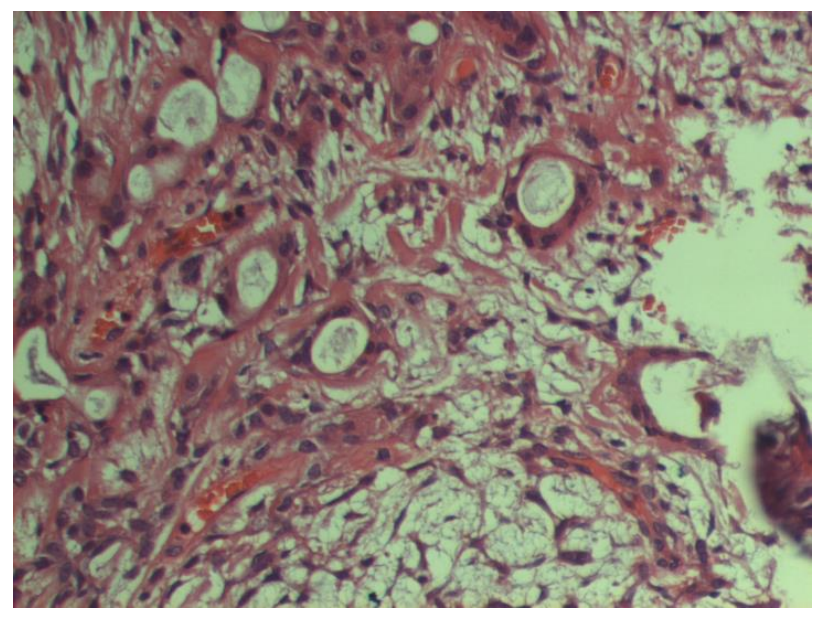

Figura 5: Imagem do corte histológico do adenoma pleomórfico evidenciando epitélio ductal e mioepitelial. (Hematoxilina \& Eosina, x40) 


\section{DISCUSSÃO}

O tratamento do adenoma pleomórfico consiste na enucleação tumoral com ou sem remoção de tecido glandular em decorrência das altas taxas de recidiva ${ }^{4}$. Segundo a literatura, a razão para a recidiva deste tumor, é sua incompleta ressecção cirúrgica trans-operatória, pois houve ruptura e/ou exposição da cápsula no procedimento ${ }^{10}$.

No presente caso clínico, foi realizada uma biópsia incisional pré-operatória com intuito do diagnóstico prévio da lesão. No entanto, segundo Smith e Komisar ${ }^{11}$, muitos cirurgiões não as realizam e assim não sabem qual patologia estão lidando. $\mathrm{O}$ julgamento clínico é o parâmetro primordial para decidir a técnica cirúrgica correta.

A escolha do tratamento pelo cirurgião varia de acordo com a localização do tumor. Setenta e sete por cento dos pacientes apresentam a lesão no lobo superficial $^{6}$. Neste relato de caso optou-se pela enucleação devido ser um procedimento rápido ${ }^{12}$, a superficialidade do tumor na glândula, a menor possibilidade de dano ao nervo facial $\mathrm{e}$ as características clínicas e imaginológicas apresentadas. Laskawi et al. ${ }^{6}$ trataram 223 pacientes com adenoma pleomórfico em glândula parótida através dos 3 tipos de cirurgia. Foram tratados com parotidectomia superficial 139 , com parotidectomia total 60 e com enucleação 14 pacientes. Um paciente, operado com parotidectomia superficial, teve recorrência após 77 meses. Um outro paciente, tratado com enucleação, teve recorrência após 131 meses de pós-operatório e nenhum caso, tratado com parotidectomia total, teve recorrência.

Tumores apresentando proporções com cerca de 4 a $6 \mathrm{~cm}$, possuem baixas recorrências. Isto pode ser explicado, pois grandes tumores necessitam de excisões mais extensas, sendo similar a parotidectomia superficial. Tumores menores apresentam crescimento multifocal de $0,5 \%$ a $23 \%$ dos casos. Isto pode ser a razão para as altas taxas de recidiva, pois deixa-se a parte substancial do parênquima do lobo superficial intacto ${ }^{10}$.

As complicações pós-operatórias encontradas são infecções, hematomas, seromas, fístulas salivares, síndrome de Frey e lesão ao nervo facial ${ }^{2,13}$. As infecções da ferida são raras e ocorrem devido a má assepsia da mesma. Os hematomas ocorrem devido a hemostasia deficiente, discrasia sanguínea, drenagem insuficiente ou compressão inadequada. Fístula salivar ou seromas são complicações raras que se manifestam após parotidectomia superficial ou excisão extracapsular. A síndrome do nervo aurículotemporal possui taxas variando de $1.14 \%$ a $40 \%{ }^{14}$. No caso apresentado, não houve complicações pósoperatórias.

Lesões ao nervo facial variam de acordo com o sítio e a severidade da lesão ${ }^{15}$. As consequências podem ser uma paralisia ou hipofunção de um ramo; hipofunção total, parcial ou paralisia completa do nervo $^{16}$. Devido a íntima relação entre o nervo facial e a glândula parótida, o risco de lesão ocorre especialmente quando a massa tumoral está muito distorcida e penetrando no parênquima da glândula parótida $^{17}$.

\section{CONCLUSÃO}

A escolha da enucleação como opção de tratamento do adenoma pleomórfico, ocorre devido ao menor índice de complicações trans e pósoperatórias, menor tempo cirúrgico, de maior simplicidade e de menor risco as estruturas adjacentes ao parênquima da glândula. Contudo, um exame clínico minucioso e exames imaginológicos associados a uma biópsia pré cirúrgica, vão permitir ao cirurgião definir qual procedimento cirúrgico é o mais indicado para o caso

\section{REFERÊNCIAS}

1. Shehata EA. Extra-capsular dissection for benign parotid tumours. Int $\mathrm{J}$ Oral Maxillofac Surg. 2010;39(2):140-44.

2. Langdon JD. Complications of parotid gland surgery. J Maxillofac Surg. 1984;12(5):225-29.

3. Silva DN, Guimarães KB, Ferraro-Bezerra $M$, Heitz C. Enucleação de adenoma pleomórfico: considerações terapêuticas e relato de caso. . Rev Cir Traumatol BucoMaxilo-Fac. 2007;7(4):25-30.

4. Chang EZ, Lee WC. Surgical treatment of pleomorphic adenoma of the parotid gland: report of 110 cases. J Oral Maxillofac Surg. 1985;43(9):680-82.

5. Westra WH. The surgical pathology of salivary gland neoplasms. Otolaryngol Clin North Am. 1999;32(5):919-43.

6. Laskawi R, Schott T, Mirzaie-Petri M, Schroeder M. Surgical management of pleomorphic adenomas of the parotid gland: a follow-up study of three methods. J Oral Maxillofac Surg. 1996;54(10):1176-79.

7. Gnepp DR. Malignant mixed tumors of the salivary glands: a review. Pathol Annu. 1993;28 Pt 1:279-328.

8. Piekarski J, Nejc D, Szymczak W, Wronski K, Jeziorski A. Results of extracapsular dissection of pleomorphic adenoma of parotid gland. J Oral Maxillofac Surg. 2004;62(10):1198-202.

9. Henriksson G, Westrin KM, Carlsöö B, Silfverswärd C. Recurrent primary pleomorphic adenomas of salivary gland origin: intrasurgical rupture, histopathologic features, and pseudopodia. Cancer. 1998;82(4):617-20.

10. Martis C. Parotid benign tumors: comments on surgical treatment of 263 cases. Int J Oral Surg. 1983;12(4):211-20.

11. Smith SL, Komisar A. Limited parotidectomy: the 
role of extracapsular dissection in parotid gland neoplasms. Laryngoscope. 2007;117(7):1163-67.

12. Dallera P, Marchetti C, Campobassi A. Local capsular dissection of parotid pleomorphic adenomas. Int $\mathbf{J}$ Oral Maxillofac Surg. 1993;22(3):154-57.

13. Giannone N, Lo Muzio L, Politi M. Extracapsular lumpectomy and SMAS flap for benign parotid tumours: an early outcome in a small number of cases on Frey's syndrome and facial nerve dysfunction. J Craniomaxillofac Surg. 2008;36(4):239-43.

14. Yoel J. Pathology and surgery ofthe salivary glands. Charles C. Thomas, Springfield; 1975, pp. 406-431.

15. Leverstein $H$, van der Wal JE, Tiwari RM, van der Waal I, Snow GB. Surgical management of 246 previously untreated pleomorphic adenomas of the parotid gland. Br J Surg. 1997;84(3):399-403.

16. Brusati R, Bozzetti A, Chiapasco M, Facil Nerve and Parotid Surgery. J. Cranio-Max. Fac. Surg. 1987; 15:278-80.

17. Touquet R, Mackenzie IJ, Carruth JA. Management of the parotid pleomorphic adenoma, the problem of exposing tumour tissue at operation. The logical pursuit of treatment policies. $\mathrm{Br} \quad \mathrm{J}$ Oral Maxillofac Surg. 1990;28(6):404-8.

\section{CONFLITO DE INTERESSES}

Os autores declaram não haver conflitos de interesse

\section{AUTOR PARA CORRESPONDENCIA}

\section{Rodrigo dos Santos Pereira}

Rua Hildegardo de Noronha 1514, apartamento 303 - Alto 25961-260 Teresópolis - RJ, Brasil

E-mail: dr.pereira@live.com
Submetido em 17/10/2019

Aceito em 22/04/2020 